\title{
Robust Gain-Scheduled Fault Tolerant Control For A Transport Aircraft
}

\author{
Jong-Yeob Shin and Irene Gregory
}

\begin{abstract}
This paper presents an application of robust gainscheduled control concepts using a linear parameter-varying (LPV) control synthesis method to design fault tolerant controllers for a civil transport aircraft. To apply the robust LPV control synthesis method, the nonlinear dynamics must be represented by an LPV model, which is developed using the function substitution method over the entire flight envelope. The developed LPV model associated with the aerodynamic coefficient uncertainties represents nonlinear dynamics including those outside the equilibrium manifold. Passive and active fault tolerant controllers (FTC) are designed for the longitudinal dynamics of the Boeing 747-100/200 aircraft in the presence of elevator failure. Both FTC laws are evaluated in the full nonlinear aircraft simulation in the presence of the elevator fault and the results are compared to show "pros" and "cons" of each control law.
\end{abstract}

\section{INTRODUCTION}

Aircraft loss-of-control (LOC) accidents [1] comprise the largest and most fatal aircraft accident category across all civil transport classes, and can result from a large array of causal and contributing factors (e.g., system and component failures, control system impairment or damage, inclement weather, inappropriate pilot inputs, etc.) occurring either individually or in combination. Research [2]-[5] into the characterization of the aircraft LOC phenomenon as well as LOC prevention and recovery system technologies is being conducted by NASA as part of its Aviation Safety Program (AvSP). Thus, fault tolerant control (FTC) for a transport aircraft plays an important role in preventing LOC aircraft accidents due to control upset or failure.

The concept of FTC has been used for various applications [6]-[8] including flight control. In general, the FTC systems can be categorized into two classes: passive and active [9]. A passive FTC law is designed with the consideration of a set of pre-modeled failures as uncertain dynamics. Thus, the gains are not recalculated based on faults but are robust enough to maintain closed-loop system stability in the presence of faults at the cost of conservative nominal performance. Hence, a system with a passive FTC law is not vulnerable to a fault detection false alarm, to fault detection time delay or to incorrect fault identification.

An active FTC law recomputes its gains due to faults to maintain system performance and stability. It is less conservative than a passive FTC law and achieves better

This work was supported by NASA under NCC-1-02043

J-Y. Shin is a senior staff scientist at the National Institute of Aerospace, 100 Expolration Way, Hampton, VA 23666, shinjy@ nianet.org

I. Gregory is a senior research engineer at the NASA Langley Research Center, Hampton,VA 23683, i.m.gregory@larc.nasa.gov performance. Fault detection and isolation (FDI) filters are, however, required to implement an active FTC law since estimated fault parameters are used to recalculate the gains. Fault parameter estimation effects on performance and even stability of the integrated system must be considered in a validation and verification process [10]. To consider the fault parameter estimation effect on the closed-loop system, fault detection times (fault estimation time delay) are assumed and are simulated with the full nonlinear closed-loop system. Based on the simulation results, acceptable fault detection time delay is estimated for maintaining closed-loop stability.

One approach to designing a FTC law is a linear parameter varying (LPV) control synthesis method with pre-defined fault models using linear matrix inequality (LMI) optimization. Incorporating the benefits of the robust control concept developed for an LTI system, the LPV synthesis method has been successfully applied to various nonlinear problems: F16 aircraft [11], turbofan engines [12], an inverted pendulum [13], etc. In Refs. [2], [14], a robust LPV synthesis method has been introduced to reduce conservatism induced by the structured uncertainty block. In Ref. [2], this method was applied to designing a FTC system of the HiMAT aircraft described by a linearized model at a trim point.

Ref. [15] presents a reconfigurable LPV control design for a Boeing 747-100/200 aircraft using the conventional LPV synthesis method in the presence of an elevator fault with unmodeled dynamics. In this paper, the robust LPV synthesis method is applied to the design of a FTC law for the longitudinal motion of the aircraft that includes the full nonlinear dynamics and uncertain aerodynamic coefficients. The magnitude of uncertainty of aerodynamic coefficients and the moment of inertia term $\left(I_{y y}\right)$ of the aircraft are pre-defined and are considered in the control synthesis procedure. Aerodynamic coefficients in tabulated data are fit as polynomial functions to generate a LPV model of the aircraft. The fitting errors are integrated into the uncertainties of aerodynamic coefficients.

A FTC law has to be robust to the given uncertainty for reliability of the implemented control system. In order to design a robust LPV-FTC law of the transport aircraft, the aerodynamic uncertainties are explicitly considered in the control design procedure. In general, a robust control design problem cannot be formulated as a linear matrix inequality that finds the global solution. The problem is generally solved by an iteration method (e.g. D-K iteration [16]). In this paper, the robust LPV synthesis method in Ref. [2] is used, which is an iteration method for solving two LMI optimization problems to reduce the conservatism introduced 
by the structured uncertainty block.

To design passive and active FTC laws for the longitudinal motion of the aircraft using the robust LPV synthesis method, the nonlinear dynamics of the aircraft with uncertain aerodynamic coefficients have to be represented over the given flight envelope by a quasi-LPV model. In this paper, the function substitution method is used to develop the quasiLPV model of the longitudinal motion of the aircraft. This method can convert the nonlinear dynamics into a quasi-LPV form over the non-trim region while preserving the stability of the original nonlinear system [17].

In this paper, both FTC laws are simulated with full nonlinear dynamics in the presence of elevator fault and the results are compared. Moreover, the closed-loop system with the active FTC law is simulated with different fault detection time delay intervals. In the example, the time delay makes the closed-loop system uncontrollable. This paper highlights the issue of a reliable fault detection time delay interval, in which the controller can preserve stability. Calculating a reliable fault detection time interval is an interesting problem. However, it will be considered as a future research topic.

This paper is organized as follows. Section 2 describes the function substitution method and the nonlinear longitudinal motion of the Boeing 747-100/200 aircraft. Using the dynamics substitution method, the nonlinear longitudinal dynamics are rewritten as a quasi-LPV model with an uncertainty block which represents real parameter uncertainties of the aerodynamic coefficients. Section 3 defines the control objectives for the longitudinal dynamics and the robust LPV control synthesis framework. Section 4 presents the simulation results for elevator faults with a passive LPVFTC law, an active LPV-FTC law, and a LPV law for healthy elevator condition. Conclusions are presented in Section 5.

\section{LPV MODEL REPRESENTATION}

\section{A. Function Substitution Method}

The function substitution method, introduced in Ref. [18], has been used to develop a quasi-LPV model for nonlinear dynamics found in Refs. [11], [17], [19], [20]. The nonlinear longitudinal dynamics of a Boeing 747-100/200 aircraft can be described by the following nonlinear model:

$$
\left[\begin{array}{l}
\dot{x}_{1} \\
\dot{x}_{2}
\end{array}\right]=A_{n l}\left(x_{1}\right)\left[\begin{array}{l}
x_{1} \\
x_{2}
\end{array}\right]+B_{n l}\left(x_{1}\right) u+l_{n l}\left(x_{1}\right)
$$

with a state vector $x^{T}=\left[\begin{array}{ll}x_{1}^{T} & x_{2}^{T}\end{array}\right] \in \mathcal{R}^{n_{x}}$ and a control vector $u \in \mathcal{R}^{n_{u}}$. The function $A_{n l}\left(x_{1}\right), B_{n l}\left(x_{1}\right)$ and $l_{n l}\left(x_{1}\right)$ are continuous mapping functions: $\mathcal{R}^{n_{x_{1}}} \mapsto$ $\mathcal{R}^{n_{x} \times n_{x}}, \mathcal{R}^{n_{x_{1}}} \mapsto \mathcal{R}^{n_{x} \times n_{u}}$ and $\mathcal{R}^{n_{x_{1}}} \mapsto \mathcal{R}^{n_{x}}$. Note that the part $\left(x_{2}\right)$ of the state vector and the control vector enter the nonlinear dynamics in a linearly affine manner.

Assume that there is a reference point $x_{r}^{T}=\left[\begin{array}{ll}x_{1 r}^{T} & x_{2 r}^{T}\end{array}\right]$ which satisfies a trim condition. A nonlinear model in Eq. (1) is rewritten as

$$
\left[\begin{array}{c}
\dot{\tilde{x}}_{1} \\
\dot{\tilde{x}}_{2}
\end{array}\right]=A_{n l}\left(x_{1}\right)\left[\begin{array}{l}
\tilde{x}_{1} \\
\tilde{x}_{2}
\end{array}\right]+B_{n l}\left(x_{1}\right) \tilde{u}+h\left(x_{1}\right)
$$

where

$$
h\left(x_{1}\right)=A_{n l}\left(x_{1}\right)\left[\begin{array}{l}
x_{1 r} \\
x_{2 r}
\end{array}\right]+B_{n l}\left(x_{1}\right) u_{r}+l_{n l}\left(x_{1}\right)
$$

and $\tilde{x}=x-x_{r}, \tilde{u}=u-u_{r}$. To convert the nonlinear dynamics into a quasi-LPV form, the function $h\left(x_{1}\right)$ is replaced by $H\left(x_{1}\right) \tilde{x}_{1}$ where $H\left(x_{1}\right)$ is a mapping function: $\mathcal{R}^{n_{x_{1}}} \mapsto \mathcal{R}^{n_{x} \times n_{x_{1}}}$. A quasi-LPV model is

$$
\left[\begin{array}{c}
\dot{\tilde{x}}_{1} \\
\dot{\tilde{x}}_{2}
\end{array}\right]=\left[A_{n l}\left(x_{1}\right)+\left[H\left(x_{1}\right) \mid 0_{n_{x} \times n_{x_{2}}}\right]\right]\left[\begin{array}{c}
\tilde{x}_{1} \\
\tilde{x}_{2}
\end{array}\right]+B_{n l}\left(x_{1}\right) \tilde{u}
$$

where $0_{n_{x} \times n_{x_{2}}}$ denotes a zero matrix with the dimension of $n_{x}$ by $n_{x_{2}}$. The key to the function substitution method is to construct the matrix function $H\left(x_{1}\right)$. The number of possible solutions satisfying the equality condition, $h\left(x_{1}\right)=$ $H\left(x_{1}\right) \tilde{x}_{1}$, is infinite because the equality constraint is an under-determined problem.

In Ref. [18], the matrix function $H\left(x_{1}\right)$ is calculated as $h\left(x_{1}\right) / \tilde{x}_{1}$. Therefore, there is singularity at the point: $x_{1}=x_{1 r}$. In Refs. [11], [19], [20], the matrix function is calculated by using linear optimization formulated at all given grid points with smoothness constraints. The reference point is not chosen as a grid point because of the singularity. In Ref. [17], the function value at $x_{1}=x_{1 r}$ is replaced by the limit value of $\lim _{x_{1} \rightarrow x_{1 r}} \frac{h\left(x_{1}\right)}{\tilde{x}_{r}}$ to remove the singularity. In the process of linear optimization in Refs. [11], [17][20], the aerodynamic coefficients of the nonlinear aircraft dynamics are evaluated at all grid points from the tabulated data. However, the methods in Refs. [11], [17]-[20] are not guaranteed to satisfy the equality condition between grid points. In Ref. [17], the preservation of stability of the original nonlinear dynamics was taken into consideration during the process of constructing the matrix function $H\left(x_{1}\right)$.

In this paper, the nonlinear function $h\left(x_{1}\right)$ is rewritten as a polynomial function of $x_{1}$ with the aerodynamic coefficients expressed as polynomials over the given flight envelope. To construct the matrix $H\left(x_{1}\right)$, the function $h\left(x_{1}\right)$ is decomposed into coefficients of $\tilde{x}_{1}$ and each coefficient is collected into the matrix form. For example, suppose

$$
h\left(x_{1}\right)=h\left(\tilde{x}_{1}+x_{1 r}\right)=\left[\begin{array}{c}
a_{1} \tilde{\alpha}+a_{2} \tilde{\alpha} \tilde{V}^{2} \\
b_{1} \tilde{V}+b_{2} \tilde{\alpha} \tilde{V}
\end{array}\right]
$$

where $a_{i}$ and $b_{i}$ are constant. The $h\left(x_{1}\right)$ can be rewritten as

$$
\begin{aligned}
h\left(x_{1}\right) & =H\left(x_{1}\right) \tilde{x}_{1}=H\left(\tilde{x}_{1}+x_{1 r}\right) \tilde{x}_{1} \\
& =\left[\begin{array}{cc}
a_{1}+a_{2} p_{1} \tilde{V}^{2} & a_{2}\left(1-p_{1}\right) \tilde{\alpha} \tilde{V} \\
b_{2} p_{2} \tilde{V} & b_{1}+b_{2}\left(1-p_{2}\right) \tilde{\alpha}
\end{array}\right]\left[\begin{array}{c}
\tilde{\alpha} \\
\tilde{V}
\end{array}\right]
\end{aligned}
$$

The values of $p_{1}$ and $p_{2}$ are chosen to preserve the stability of the original nonlinear model [17]. These underdetermined conditions can be solved numerically, as was done in previous work [17]. Here however, the equations are solved symbolically. The benefits of the symbolic solution are:1) simplification that avoids the need for a linear optimization problem with the smoothness condition, and 2) validation of the quasi-LPV model between grid points, including computed fitting errors. Moreover, the fitting errors can be integrated with the uncertainty block in the quasi-LPV model. 
TABLE I

THE UNCERTAIN AERODYNAMIC COEFFICIENTS

\begin{tabular}{|c|c|c|c|}
\hline \hline coefficient & uncertain range $\left(\delta_{a}\right)$ & coefficient & uncertain range $\left(\delta_{a}\right)$ \\
\hline$C_{D_{M}}$ & {$\left[\begin{array}{ll}-0.01 & 0.01\end{array}\right]$} & $C_{L_{q}}$ & {$\left[\begin{array}{ll}-0.15 & 0.20\end{array}\right]$} \\
$C_{L_{b}}$ & {$\left[\begin{array}{ll}-0.025 & 0.025\end{array}\right]$} & $C_{m_{s}}$ & {$\left[\begin{array}{ll}-0.07 & 0.03\end{array}\right]$} \\
$C_{m_{b}}$ & {$\left[\begin{array}{ll}-0.005 & 0.005\end{array}\right]$} & $C_{m_{e}}$ & {$\left[\begin{array}{ll}-1, & 1\end{array}\right] \times 10^{-4}$} \\
$C_{L_{e}}$ & {$\left[\begin{array}{ll}-6 & 6\end{array} 0^{-6}\right.$} & $C_{m_{q}}$ & {$\left[\begin{array}{lll}-0.83 & 0.6\end{array}\right]$} \\
\hline \hline
\end{tabular}

\section{B. Longitudinal dynamics of a transport aircraft}

The nonlinear longitudinal dynamics of a transport aircraft (Boeing 747-100/200 aircraft) are taken from Refs. [20][22]. The longitudinal motion states and controls are $x=$ $[\alpha, q, V, \theta]^{T}$ (angle of attack (deg), pitch rate $(\mathrm{deg} / \mathrm{sec}$ ), velocity $(\mathrm{m} / \mathrm{sec})$, pitch angle $(\mathrm{deg}))$ and $u=\left[\delta_{e}, \delta_{s}, T\right]^{T}$ (elevator deflection (deg), horizontal stabilizer deflection (deg) and thrust $(\mathrm{N})$ ), respectively. The nonlinear longitudinal dynamics are documented in Refs. [21], [22]. In this paper, the flight envelope for the longitudinal dynamics is defined as $\alpha \in\left[\begin{array}{ll}-2 & 10\end{array}\right](\mathrm{deg})$ and $V \in\left[\begin{array}{ll}100 & 250\end{array}\right](\mathrm{m} / \mathrm{sec})$ at an altitude of $7000 \mathrm{~m}$. Aerodynamic coefficients and their derivatives are fit into a polynomial function form such that

$$
a=\left[\begin{array}{llll}
\alpha^{n} & \alpha^{(n-1)} & \cdots & 1
\end{array}\right] C_{a}\left[\begin{array}{llll}
V^{m} & V^{(m-1)} & \cdots & 1
\end{array}\right]^{T}+\delta_{a}
$$

where $a$ is an aerodynamic coefficient, $C_{a}$ is a coefficient matrix and $\delta_{a}$ is a fitting error over the flight envelope. The aerodynamic coefficients and their uncertainties are given in Table I. The drag coefficient at fixed mach number $\left(C_{D_{M}}\right)$, the lift coefficient at zero stabilizer angle $\left(C_{L_{b}}\right)$, the pitch moment coefficient at zero stabilizer angle $\left(C_{m_{b}}\right)$, the lift coefficient derivative due to elevator deflection $\left(C_{L_{e}}\right)$, and the pitching moment coefficient derivative due to elevator deflection $\left(C_{m_{e}}\right)$ are functions of angle of attack and velocity, and the lift coefficient derivative due to pitch rate $\left(C_{L_{q}}\right)$, the pitching moment coefficient derivative due to stabilizer deflection $\left(C_{m_{s}}\right)$, and the pitching moment coefficient derivative due to pitch rate $\left(C_{m_{q}}\right)$ are constant over the flight envelope. The detailed polynomial fit functions are omitted due to limited space. Note that the aerodynamic coefficients are evaluated in the full nonlinear simulation with the tabulated data. The polynomial fit aerodynamic coefficients are only used for the quasi-LPV model development.

\section{LPV model with real parameter uncertainties}

Using the symbolic tool in MATLAB and the function substitution method, the nonlinear longitudinal model can be rewritten as

$$
\begin{gathered}
{\left[\begin{array}{c}
\dot{x} \\
z_{p} \\
y
\end{array}\right]=\left[\begin{array}{ccc}
A(\alpha, V) & B_{w}(\alpha, V) & B_{u}(\alpha, V) \\
C_{z}(\alpha, V) & D_{z w}(\alpha, V) & D_{z u}(\alpha, V) \\
C_{y}(\alpha, V) & D_{y w}(\alpha, V) & D_{y u}(\alpha, V)
\end{array}\right]\left[\begin{array}{c}
x \\
w_{p} \\
u
\end{array}\right]} \\
w_{p}=\Delta_{p} z_{p}
\end{gathered}
$$

where $\Delta_{p}$ represents real parameter uncertainties of the aerodynamic coefficients. With the assumption that the moment of inertia $\left(I_{y y}\right)$ uncertainty is 5 percent of nominal value (45278000 Kg m $\left.\mathrm{K}^{2}\right)$, the $\Delta_{p}$ is

$$
\begin{aligned}
& \Delta_{p}=\operatorname{diag}\left(\left[\delta_{C_{D_{M}}}, \delta_{C_{L_{b}}}, \delta_{C_{m_{b}}}, \delta_{C_{L_{e}}},\right.\right. \\
& \left.\left.\delta_{C_{L_{q}}}, \delta_{C_{m_{s}}}, \delta_{C_{m_{q}}}, \delta_{C_{m_{e}}}, \delta_{I_{y y}}\right)\right]
\end{aligned}
$$

Note that the 9 real uncertainty parameters are normalized such that $\left|\Delta_{p}\right| \leq 1$.

For comparison between the linearized model and the quasi-LPV model, an extreme flight maneuver (shown in Fig. 1) is simulated for the elevator and thrust input signals to show which model can best capture the original nonlinear model. Time responses for each model are shown in Fig. 1. Note that the linearized model (LTI) is obtained using Jacobian linearization around a trim point $\left(x_{t} \approx[1.1(\mathrm{deg}), \quad 0, \quad 228(\mathrm{~m} / \mathrm{s}), \quad 1.1(\mathrm{deg})], \quad u_{t} \approx\right.$ $[-2(\mathrm{deg}), 1.3(\mathrm{deg}), 43000(N)])$ at altitude of $7000 \mathrm{~m}$. It is observed from Fig. 1 that the quasi-LPV model can capture the nonlinear aspects of the original model better than the linearized model.
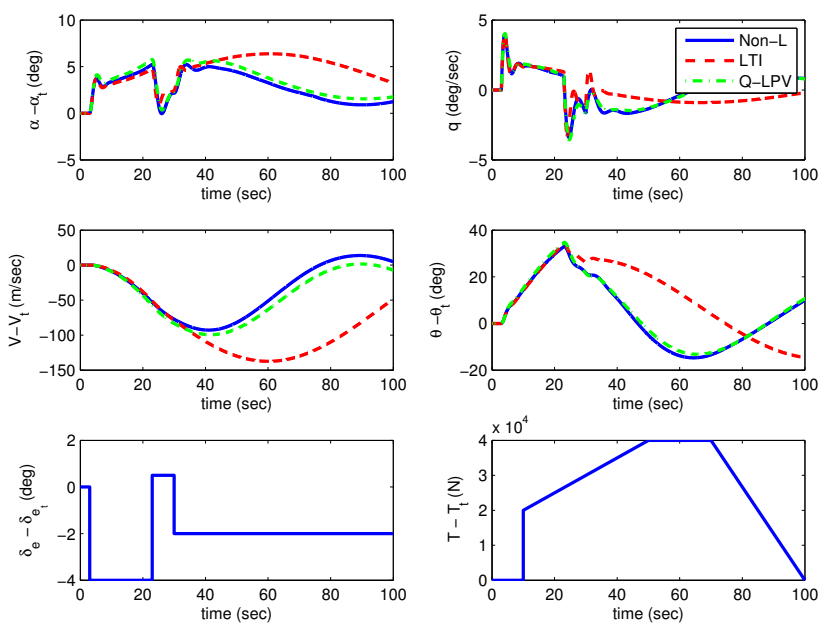

Fig. 1. The simulation results of the nonlinear model (Non-L), the quasiLPV model (Q-LPV), and the linearized model (LTI)

\section{Robust LPV FTC CONTROL SYNTHESIS}

\section{A. Control Objectives}

The control objectives for the transport aircraft are to obtain good tracking on flight path angle $(\gamma)$ and velocity commands in the presence of parameter uncertainties and an elevator fault. The elevator fault is modeled as zero controllability in the elevator channel. The elevator and stabilizer actuator dynamics are modeled as

$$
\delta_{e}=\rho_{f} \frac{37}{s+37} \delta_{e_{c m d}}, \quad \delta_{s}=\left(1-\rho_{f}\right) \frac{0.5}{s+0.5} \delta_{s_{c m d}}
$$

with the fault parameter $\left(\rho_{f}\right)$ which can vary from 0 (failure case) to 1 (healthy case). Here $\delta_{e_{c m d}}$ and $\delta_{s_{c m d}}$ are actuator command signals from a control law. The engine is modeled as the first order transfer function $\frac{0.5}{s+0.5}$. The deflection and rate limits for the elevator and the stabilizer are $-23 \leq$ $\delta_{e} \leq 17,\left|\dot{\delta_{e}}\right| \leq 37(\mathrm{deg} / \mathrm{sec})$ and $-12 \leq \delta_{s} \leq 3,\left|\dot{\delta_{s}}\right| \leq$ $0.5(\mathrm{deg} / \mathrm{sec})$ (Refs. [15], [20], [22]). The maximum thrust 
and thrust rate are $167000 \mathrm{~N}$ and $83500 \mathrm{~N} / \mathrm{s}$, respectively. Actuator models and their limits are included in the nonlinear simulations. To formulate the control objectives into an

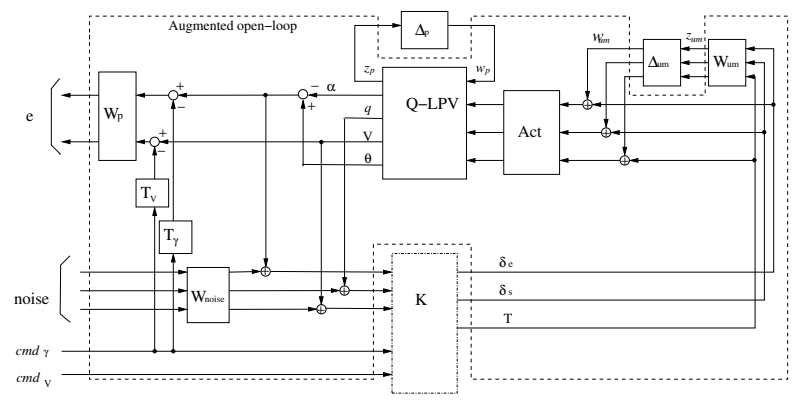

Fig. 2. The interconnection structure with a controller and weighting functions.

optimization problem of minimizing the induced- $\mathcal{L}_{2}$ norm of the closed-loop system from commands to tracking errors, the model matching framework shown in Fig. 2 is used in this paper. The desired velocity tracking model $\left(T_{V}\right)$ is chosen as the second order system $\frac{0.15^{2}}{s^{2}+0.3 s+0.15^{2}}$ from Ref. [15] with $0.15 \mathrm{rad} / \mathrm{sec}$ as the cut off frequency. The desired flight path tracking model $\left(T_{\gamma}\right)$ is chosen as the second order system $\left(\frac{0.7^{2}}{s^{2}+1.4 s+0.7^{2}}\right)$ having a cut-off frequency of $0.7 \mathrm{rad} / \mathrm{sec}$. The desired transfer functions are defined based on control designer judgment. The performance weighting function $W_{p}$ is chosen as $\operatorname{diag}\left(\left[\frac{50(s / 300+1)}{s / 0.01+1}, \frac{50(s / 100+1)}{s / 0.005+1}\right]\right)$ to penalize the tracking error at low frequency range (desired tracking within $2 \%$ of command) and to be relaxed at high frequency since unmodeled actuator dynamic uncertainty is greater than $100 \%$ at high frequency. The unmodeled dynamics $W_{u m}$ [15] of actuators are set as $\operatorname{diag}\left(\left[\frac{0.01(s / 1+1)}{s / 500+1}, \frac{0.01(s / 0.5+1)}{s / 300+1}, \frac{0.01(s / 1+1)}{s / 500+1}\right]\right)$ to represent $1 \%$ uncertainty at low frequency. The noise level $W_{\text {noise }}$ is set as $\operatorname{diag}([0.05 \mathrm{deg}, \quad 0.1 \mathrm{deg} / \mathrm{sec}, \quad 0.05 \mathrm{~m} / \mathrm{sec}])$. The

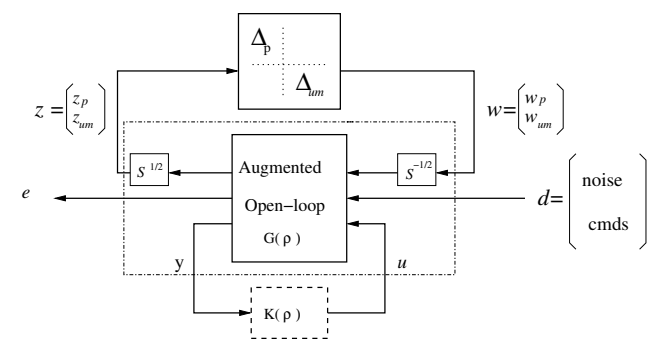

Fig. 3. The augmented open-loop with the scaling factor associated with the uncertainty block $\Delta$.

augmented open-loop system is represented by the dasheddotted box shown in Fig. 3. The augmented state vector $x$ includes longitudinal dynamics states, actuator states, and weighting function states; the vectors $z$ and $w$ are associated with the uncertainty block $\Delta, \operatorname{diag}\left(\left[\Delta_{p}, \Delta_{u m}\right]\right)$, the vector $e$ represents weighted tracking errors, and the vector $d$ represents noise inputs, flight path angle and velocity commands. Note that the command signals are treated as disturbance signals here. The scaling matrix $S$ over the uncertainty block relating the input and output signals belongs to a set $\mathcal{S}_{\Delta}$ :

$$
\mathcal{S}_{\Delta}=\left\{S: S>0, S \Delta=\Delta S, S \in \mathcal{R}^{n_{\Delta} \times n_{\Delta}}\right\} .
$$

In Fig. 3, the scheduling parameter $\rho$ represents angle of attack $\alpha$, velocity $V$, and the fault parameter $\rho_{f}$.

\section{B. Linear Matrix Inequality Optimization Formulation}

The robust LPV control synthesis problem is stated as designing an LPV controller with the scaling matrix $S$ to minimize the induced $\mathcal{L}_{2}$ norm of the closed-loop system.

$$
\min _{K, S} \gamma=\frac{\left\|\left[z^{T} e^{T}\right]^{T}\right\|_{2}}{\left\|\left[w^{T} d^{T}\right]^{T}\right\|_{2}} .
$$

This problem is a bilinear matrix inequality problem similar to a $\mathrm{D}-\mathrm{K}$ iteration problem for a linear system. In this paper, this problem is solved by an iterative procedure using the linear matrix inequality (LMI) optimization. The iteration procedure is as follows:

1) Design an LPV controller $K$ using a conventional LPV synthesis method [23], [24], with an assumption that $S=$ $I$, to minimize the induced- $\mathcal{L}_{2}$ norm index $\gamma$ over all LMI constraints evaluated at all grid points. The grid points are set as $V=\left[\begin{array}{lllll}110 & 140 & 170 & 200 & 230\end{array}\right]$ and $\rho_{f}=\left[\begin{array}{llll}0.01 & 0.3 & 0.6 & 1\end{array}\right]$.

2) Combine the designed controller $K$ and the augmented open-loop system with all weighting functions, resulting in the augmented closed-loop system given by

$$
\left[\begin{array}{c}
\dot{x}_{c l} \\
p
\end{array}\right]=\left[\begin{array}{lll}
A_{c l} & B_{1_{c l}} & B_{2_{c l}} \\
C_{c l} & D_{1_{c l}} & D_{2_{c l}}
\end{array}\right]\left[\begin{array}{c}
x_{c l} \\
w \\
d
\end{array}\right]
$$

where the vector $p$ is $\left[z^{T} e^{T}\right]$.

3) Calculate a scaling matrix $S$ solving the following LMI optimization problem. Applying the Kalman-YakubovichPopov lemma (Ref. [25]), the following LMI optimization is formulated to minimize the induced $-\mathcal{L}_{2}$ norm of the augmented closed-loop system from $d$ to $e$.

$$
\min _{P, S} \gamma_{c}
$$

subject to

$$
\left[\begin{array}{ccc}
M_{11} & P B_{1_{c l}}+C_{c l}^{T} \tilde{S} D_{1_{c l}} & P B_{2_{c l}}+C_{c l}^{T} \tilde{S} D_{1_{c l}} \\
(*) & D_{1_{c l}}^{T} \tilde{S} D_{1_{c l}}-S & D_{1_{c l}^{T}}^{T} \tilde{S} D_{2_{c l}} \\
(*) & (*) & D_{2_{c l}}^{T} D_{2_{c l}}-I
\end{array}\right]<0
$$

where

$$
\tilde{S}=\left[\begin{array}{cc}
S & 0 \\
0 & \gamma_{c} I_{n_{e} \times n_{e}}
\end{array}\right]
$$

and $M_{11}=A_{c l}^{T} P+P A_{c l}+\dot{P}+C_{c l}^{T} \tilde{S} C_{c l}$. The notation (*) denotes the symmetric component of the LMI constraint. Note that the LMI constraint in Eq. (16) can be derived from the candidate quadratic Lyapunov function $V=x^{T} P x$ and $\|e\|_{2}<\gamma_{c}\|d\|_{2}$.

4) The calculated scaling matrix $S$ is integrated with the augmented open-loop in Fig. 3. A new LPV controller $K$ is calculated based on the new augmented open-loop shown in dashed-dotted line in Fig. 3 to minimize an induced- $\mathcal{L}_{2}$ 
norm of the closed-loop system using the conventional LPV synthesis method.

5) Iterate steps 1-4 until optimal solution $\gamma$ value is converged or the designed controller provides satisfactory performance.

This iterative procedure does not guarantee convergence. Also note that this procedure may not find global solutions for $K$ and $S$. Using the iteration procedure, three LPV controllers are designed. 1) A nominal, no actuator failure, LPV controller $\left(K_{L P V_{h}}\right)$, robust to the real parameter uncertainty, is designed to minimize the performance index (induced- $\mathcal{L}_{2}$ norm). It is used as a baseline for comparison in case of an actuator failure. 2) A passive LPV FTC law $\left(K_{L P V_{p}}\right)$ is constructed in the presence of the elevator fault. 3) An active LPV FTC law $\left(K_{L P V_{a}}\right)$ is designed in the presence of the elevator fault with the assumption that the fault is accurately estimated on-line. For the healthy condition, elevator and thrust are used to control the longitudinal motion of the aircraft, while the stabilizer is used only as a trimming device.

To design a passive LPV-FTC law, elevator failure should be modeled into an LFT form. The failure parameter $\rho_{f}$ is modeled as the upper LFT form $\mathcal{F}_{u}\left(M, \delta_{\rho_{f}}\right)$, where $M=$ $\left[\begin{array}{cc}0 & \sqrt{0.5} \\ \sqrt{0.5} & 0.5\end{array}\right]$ with $\left|\delta_{\rho_{f}}\right| \leq 1$. We put the matrix $M$ in the signal path between the open-loop model and the elevator actuator in Fig. 2 to represent elevator failure as a parameter uncertainty. In the control design process for $K_{L P V_{p}}$, the real parameter uncertainty $\delta_{\rho_{f}}$ is appended to the uncertainty block shown in Fig. 3 such that $\Delta=\operatorname{diag}\left(\left[\Delta_{p}, \Delta_{u m}, \delta_{\rho_{f}}\right]\right)$. Note that the elevator failure is treated as a real parameter uncertainty in designing a passive FTC law.

In an active LPV-FTC law design, the elevator is used under the healthy condition and the stabilizer is used only in the presence of the elevator failure. The actuators are modeled as in Eq.(11) with fault parameter $\rho_{f}$. In this case, $\rho_{f}$ is one of the scheduling parameters. For all three LPV controllers, the minimized induced $\mathcal{L}_{2}$ norm index $\gamma$ is around 2.5. In this example during the iteration process, the $\gamma$ values do not change much. After 3 iterations, the iteration procedure is terminated.

\section{Simulation Results}

The designed LPV control laws are evaluated in the nonlinear dynamic simulation with commanded flight path angle under nominal and faulty conditions. For the faulty condition, the elevator is stuck at $10 \mathrm{sec}$. For all simulations, the flight path angle command is a 3 degree magnitude, 70 second duration pulse beginning at $5 \mathrm{sec}$. It is observed from the simulation results shown in Fig. 4 that all controllers achieve the desired performance under the healthy condition. Note that the passive FTC law $\left(K_{L P V_{p}}\right)$ uses both the elevator and the stabilizer even in the nominal case. The active FTC law, however, uses only the elevator under the healthy condition.

It is observed from Fig. 5 that the FTC controllers $K_{L P V_{p}}$ and $K_{L P V_{a}}$ achieve the desired performance in the presence of the elevator fault. Note that the closed-loop time responses for the controller $K_{L P V_{h}}$ show that the system is unstable in the presence of the elevator fault. The design of the active FTC law $K_{L P V_{a}}$ assumed that the fault parameter is accurately estimated with no time delay. Based on the fault parameter, the gains in the active FTC law are reconfigured to use the stabilizer instead of the faulty elevator.
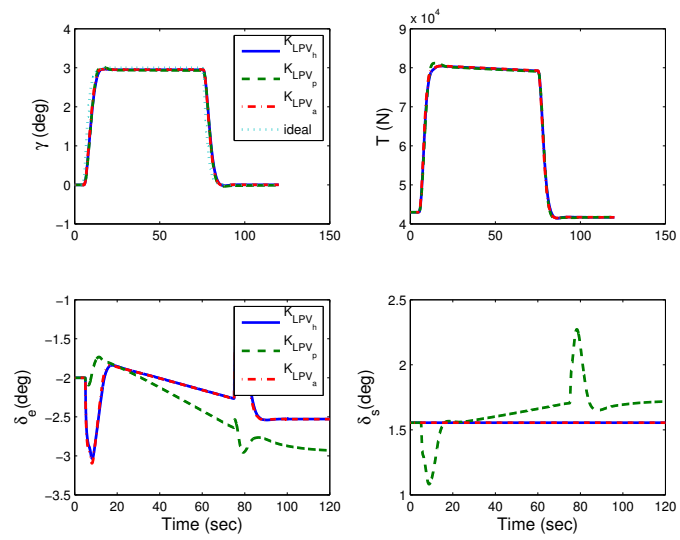

Fig. 4. The simulation results with the control laws under a healthy condition.
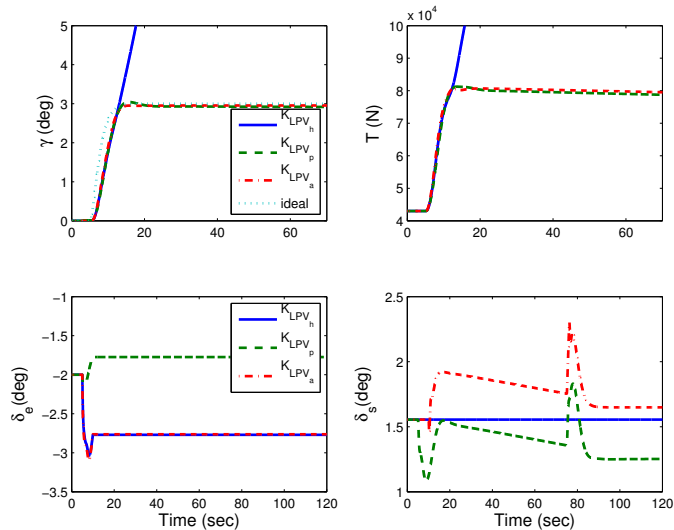

Fig. 5. The simulation results under the faulty condition: the stuck elevator at $10 \mathrm{sec}$

Although not explicitly considered in the design process, the effect of time delays in the fault detection algorithm can be examined in the nonlinear simulation. The fault detection time is defined as the time interval to detect a fault after fault occurrence. In this example, the fault detection times are assumed to be $1 \mathrm{sec}, 5 \mathrm{sec}, 10 \mathrm{sec}$ and $20 \mathrm{sec}$, respectively. For example, $5 \mathrm{sec}$ fault detection time means that when the elevator is stuck at $10 \mathrm{sec}$, the fault parameter is estimated at $15 \mathrm{sec}$. It is observed from Fig. 6 that the system remains stable for $1 \mathrm{sec}$ and $5 \mathrm{sec}$ fault detection time cases. For the $10 \mathrm{sec}$ and $20 \mathrm{sec}$ detection time cases, the system is unstable since the disturbance caused by the stuck elevator makes the system unrecoverable. It is observed from the stabilizer time responses that the stabilizer deflection rate is already saturated when the fault is detected and the controller gains recalculated. It is too late to stabilize the system using the stabilizer with its rate limit of $0.5 \mathrm{deg} / \mathrm{sec}$. For this single actuator failure case, non-zero controllability condition for 

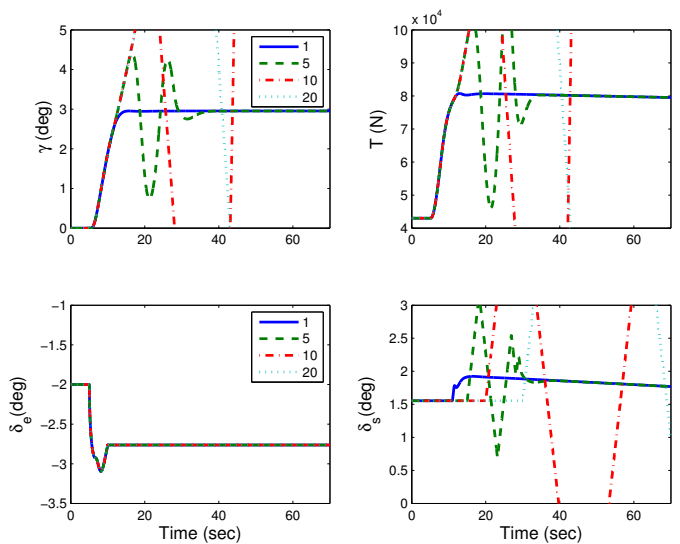

Fig. 6. The simulation results with fault detection time: $1 \mathrm{sec}, 5 \mathrm{sec}, 10$ sec and $20 \mathrm{sec}$

the active FTC law is not satisfied for the short time interval. Thus to reiterate, calculating a reliable fault detection time limit for stability and performance is still an open problem due to nonlinearity and complexity of the integrated system as well as the fault condition itself with the limited control authorities. Calculating a reliable fault detection time limit will be a future research topic.

\section{CONCLUSION}

Robust-gain scheduled control concepts have been applied to design passive and active fault tolerant control laws for a transport aircraft. In order to use the LPV synthesis method, the nonlinear model of the longitudinal aircraft dynamics is converted into a quasi-LPV form. In the LPV representation procedure, the tabulated aerodynamic coefficients are fit as polynomial functions of angle of attack and velocity. The fitting errors are integrated into the real parameter model uncertainties. The passive and active FTC laws, designed based on the quasi-LPV model, achieve the desired performance level in the presence of the prescribed elevator fault. In this example, the "pros" and "cons" of each FTC law are compared via nonlinear simulations. For the active FTC controlled system for the elevator failure, the controllability condition is not satisfied for the fault detection time delay ( $t \geq 10 \mathrm{sec}$ ). It makes the system unstable due to the limited control authority. For the passive FTC law, the controller uses both the elevator and the stabilizer for control even healthy condition.

\section{REFERENCES}

[1] Belcastro, C., Khong, T., Shin, J-Y, Balas, G., Kwatny, H., and Chang, B., "Uncertainty Modeling for Robustness Analysis of Control Upset Prevention and Recovery Systems," in AIAA Guidance, Navigation and Control Conference, AIAA 2005-6427, AIAA, 2005.

[2] Shin, J-Y., N.E. Eva, and Belcastro, C., "Adaptive Linear Parameter Varying Control Synthesis for Actuator Failure," Journal of Guidance, Control, and Dynamics, Vol. 27, Sept.-Oct. 2004, pp. 787-794.

[3] Belcastro, C. and Chang, B-C., "Uncertainty Modeling for Robustness Analysis of Failure Detection and Accommodation Systems," in IEEE American Control Conference, Vol. 6, American Control Conference, 2002, pp. 4776-4782.
[4] Jordan, T., Langford, W., and Hill, J., "Airborne Subscale Transport Aircraft Research Testbed- Aircraft Model Development," in AIAA Guidance, Navigation and Control Conference, AIAA-2005-6432, AIAA, 2005.

[5] Bailey, R., Hostetler, R., Barnes K., Belcastro, Celeste, and Belcastro, Christine, "Experimental Validation: Subscale Aircraft Ground Facilities and Integrated Test Capability," in AIAA Guidance, Navigation and Control Conference, AIAA-2005-6433, 2005, AIAA.

[6] Balle,P., Fischera, M., Fussel, D., Nells, O., and Isermann, R., "Integrated Control, Diagnosis and Reconfiguration of a Heat Exchanger,' IEEE Control Systems Magazine, 1998, pp. 52-63.

[7] Katebi, M. and Grimble, M., "Integrated Control, Guidance and Diagnosis for Reconfigurable Autonomous Underwater Vehicle Control,' International Journal of Systems Science, Vol. 30, No. 9, 1999, pp. 1021-1032.

[8] Musgrave, J., Guo, T-H., Wong, E., and Duyar, A., "Real-time Accommodation of Actuator Faults on a Reusable Rocket Engine," IEEE Transactions Control Systems Technology, No. 1, 1997, pp. 100-109.

[9] Zhang, W. and Jiang, J., "Issues on Integration of Fault Diagnosis And Reconfigurable Control in Active Fault-Tolerant Control Systems," in IFAC Symposium on Fault Detection, Supervision and Safety for Technical Processed, (Beijing, China), August-September 2006, pp. 1513-1524.

[10] Shin, J-Y, and Belcastro, C., "Performance Analysis on Fault Tolerant Control System," IEEE Transactions on Control Systems Technology, Vol. 14, No. 5, 2006, pp. 920-925.

[11] Shin, J-Y., Balas, G.J., and Kaya, M.A., "Blending Methodology of Linear Parameter Varying Control Synthesis of F-16 Aircraft System," Journal of Guidance, Control, and Dynamics, Vol. 25, No. 6, 2002, pp. 1040-1048.

[12] Balas, G., Ryan, J., Shin, J-Y., and Garrard, W., "A New Technique for Design of Controllers for Turbofan Engines," in AIAA 34th Join Propulsion Conference, (Cleveland, OH), 1998, pp. 1-6. AIAA-983751 .

[13] Kajiwara, H., Apkarian, P., and Gahinet, P., "LPV Techniques for Control of an Inverted Pendulum," in IEEE Control Systems, Feb. 1999, pp. 44-54.

[14] Apkarian, P. and Adams, R., "Advanced Gain-Scheduling Techniques for Uncertain Systems," IEEE Transactions on Control Systems Technology, Vol. 6, No. 1, 1998, pp. 21-32.

[15] Ganguli, S., Marcos, A., and Balas, G., "Reconfigurable LPV Control Design for Boeing 747-100/200 Longitudinal Axis," in Proceedings of the American Control Conference, (Anchorage, AK), May 2002. pp. 3612-3617, American Control Conference.

[16] Balas, G., Doyle, J., Glover, K., and Packard, A., $\mu$ Analysis and Synthesis Toolbox. Natick, MA: The Mathworks, Inc., 1995.

[17] Shin, J-Y., "Quasi-Linear Parameter Varying Representation of General Aircraft Dynamics Over A Non-Trim Region," National Institute of Aerospace, , Hampton, VA 23666, 2005. NASA/CR-2005213926,NIA Report No. 2005-08.

[18] Tan, W., "Applications of Linear Parameter-Varying Control Theory," Master's thesis, Department of Mechanical Engineering, University of California at Berkeley, 1997.

[19] Shin, J-Y., Worst-case Analysis and Linear Parameter Varying Control of Aerospace System. PhD thesis, Department of Aerospace Engineering and Mechanics, University of Minnesota, 2000.

[20] Marcos, A. and Balas, G., "Linear Parameter Varying Modeling of the Boeing 747-100/200 Longitudinal Motion," in AIAA Guidance, Navigation and Control Conference, AIAA-01-4347, American Institute of Aeronautics and Astronautics, (Montreal, Canada), Aug. 2001.

[21] Szaszi, I., Marcos, A., Balas, G., and Bokor, J., "Linear ParameterVarying Detection Filter Design for a Boeing 747-100/200 Aircraft," Journal of Guidance, Control, and Dynamics, Vol. 28, No. 3, 2005 , pp. 461-470.

[22] Shin, J-Y., Belcastro, C., and Khong, T., "Closed-Loop Evaluation of An Integrated Failure Identification And Fault Tolerant Control System for A Transport Aircraft," in AIAA Guidance, Navigation and Control Conference, AIAA-2006-6310, (Keystone, CO), 2006, AIAA.

[23] Becker, G., Quadratic Stability and Performance of Linear Parameter Dependent Systems. PhD thesis, Department of Engineering, University of California, Berkeley, 1993.

[24] Wu, F., Control of Linear Parameter Varying Systems. PhD thesis, Department of Mechanical Engineering, University of California, Berkeley, 1995.

[25] Dullerud, G. and Paganini, F., A Course in Robust Control Theory, A Convex Approach. Berlin: Applied Mathematics, Springer, 2000. Ch. 7. 